\title{
Factors predicting uptake of long-acting reversible methods of contraception among women presenting for abortion
}

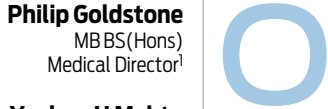
verall, Australia's population is one of the healthiest in the world. Nevertheless, the country has relatively high levels of sexual and reproductive ill health. ${ }^{1}$ It is estimated that $50 \%$ of Australian women have had an unintended pregnancy during their reproductive lives, ${ }^{2}$ with about 80000 abortions occurring annually. ${ }^{3}$ For several years, there has been growing national and international recognition that a key way to reduce unintended pregnancies is to encourage women to use more effective and less userdependent methods, specifically to increase the uptake of long-acting reversible contraceptive (LARC) methods. ${ }^{4-7}$ LARC methods include progestogen injections, progestogen-only implants, and hormonal and copper intrauterine contraceptive (IUC) methods. ${ }^{5}$ Of the LARC methods, IUC and implants are the most effective, and have the potential to reduce unintended pregnancy by 20 -fold compared with women using contraceptive pills, patches or rings. ${ }^{8}$ Despite this, less than $7 \%$ of women in Australia use an IUC or implant, ${ }^{9}$ and discussion of LARC methods features in only $15.4 \%$ of general practice contraception consultations. ${ }^{10}$

Among women seeking abortion, several studies have found that postabortion uptake of IUC or implants is associated with a significantly reduced likelihood of having a subsequent repeat abortion compared with women using the combined oral contraceptive pill.11-14 Thus, in addition to providing safe pregnancy termination services, it is now widely recommended that abortion clinics should provide high-quality counselling about future methods of contraception and, in particular, providers should communicate the greater effectiveness of LARC methods. ${ }^{15,16}$ Ideally the chosen method of contraception should be initiated immediately after abortion; ${ }^{15}$ an important

Abstract

Objective: To examine the uptake of long-acting reversible contraceptive (LARC) methods after abortion among women seeking abortions through a major Australian abortion provider.

Design and setting: Cross-sectional study of women's post-abortion contraceptive choices, conducted at Marie Stopes International clinics across Australia between 1 September and 31 December 2012.

Participants: 7267 of 9477 women (76.7\%) presenting during the study period had data collection forms completed. Analysis was based on the 6348 women with completed demographic details.

Main outcome measures: Uptake and immediate provision of LARC contraception after abortion.

Results: Just over a quarter of women (1742; 27.4\%) chose a LARC method for use after abortion. Of those choosing a LARC, immediate provision occurred in $71.1 \%$. Compared with women aged $20-24$ years, those aged $16-19$ years were less likely to have immediate LARC insertion and those over 30 more likely. Women in the lowest socioeconomic quintile were the least likely to leave the service with their chosen LARC in place compared with those in higher quintiles. Immediate LARC provision occurred more often after surgical abortion compared with medical abortion (1034 [74.4\%] v 204 [60.0\%]; $P<0.001$ ).

Conclusion: Among women who opted for a LARC method after abortion, immediate provision was less likely to occur in women aged under 30 years, less likely as their level of disadvantage increased and more likely after surgical abortion compared with medical abortion. Public health policy needs to facilitate access to LARC methods after abortion so that more women are able to avoid a further unintended pregnancy.

issue given that up to $83 \%$ of abortion clients ovulate within the first cycle. ${ }^{17}$ Immediate provision can occur at the time of a surgical abortion, as IUC methods and implants can be safely inserted or administered at the end of the procedure. ${ }^{18}$ After medical abortion IUC methods can be initiated as early as 5-9 days after abortion, once the practitioner is reasonably certain the woman is no longer pregnant. ${ }^{19}$ Implants can be inserted earlier than this. ${ }^{6}$

The not-for-profit, non-government organisation Marie Stopes International (MSI) is the largest provider of abortion services in Australia and operates in all states and territories except Tasmania, South Australia and Northern Territory. While the actual number of abortions in Australia is unknown, MSI estimates that in the states and territories where it operates, around a third of all abortions are undertaken in its centres, comprising about one-quarter of all abortions across Australia.
In this study, we aimed to determine the extent to which MSI providers were ensuring women were leaving the abortion service with a plan for contraception, what proportion stated their intent to use either an IUC or implant LARC method and of those how many received immediate provision of their nominated method. We also sought to explore the sociodemographic and reproductive health factors associated with immediate provision of LARC.

\section{Methods}

We conducted a large, prospective, multicentre cross-sectional study through MSI Australia (MSIA) clinics, the largest single provider of abortion in Australia. All 16 of the provider's centres across Australia were included. Between 1 September and 31 December in 2012, MSIA staff documented the contraceptive choices of women who had attended for a termination of pregnancy. The data 
1 Demographic and reproductive characteristics of 6348 women who had an abortion at a Marie Stopes International Australia clinic, 1 September - 31 December 2012

\begin{tabular}{|c|c|}
\hline & No. (\%) \\
\hline \multicolumn{2}{|l|}{ Age group (years) } \\
\hline Under 16 & $45(0.7 \%)$ \\
\hline 16-19 & $698(11.0 \%)$ \\
\hline $20-24$ & $1727(27.2 \%)$ \\
\hline 25-29 & $1510(23.8 \%)$ \\
\hline 30-39 & $1923(30.3 \%)$ \\
\hline 40 and over & $445(7.0 \%)$ \\
\hline \multicolumn{2}{|l|}{ Relationship status } \\
\hline Single & $2742(43.2 \%)$ \\
\hline Partnered & $3606(56.8 \%)$ \\
\hline \multicolumn{2}{|l|}{ Birthplace } \\
\hline Australia & $4083(64.3 \%)$ \\
\hline Other Pacific nation & $423(6.7 \%)$ \\
\hline Asia & $1117(17.6 \%)$ \\
\hline Africa & $161(2.5 \%)$ \\
\hline America (North and South) & $77(1.2 \%)$ \\
\hline Europe & $327(5.2 \%)$ \\
\hline Middle East & $160(2.5 \%)$ \\
\hline \multicolumn{2}{|c|}{ Socio-Economic Index for Areas quintile } \\
\hline 1 (most disadvantaged) & $993(15.6 \%)$ \\
\hline 2 & $883(13.9 \%)$ \\
\hline 3 & $1486(23.4 \%)$ \\
\hline 4 & $1236(19.5 \%)$ \\
\hline 5 (least disadvantaged) & $1750(27.6 \%)$ \\
\hline \multicolumn{2}{|l|}{ Number of children } \\
\hline 0 & $3187(50.2 \%)$ \\
\hline 1 & $1136(17.9 \%)$ \\
\hline 2 & $1280(20.2 \%)$ \\
\hline 3 & $490(7.7 \%)$ \\
\hline 4 or more & $255(4.0 \%)$ \\
\hline \multicolumn{2}{|c|}{ Number of previous terminations } \\
\hline 0 & $3933(62.0 \%)$ \\
\hline 1 & $1690(26.6 \%)$ \\
\hline 2 & $506(8.0 \%)$ \\
\hline 3 & $148(2.3 \%)$ \\
\hline 4 or more & $71(1.1 \%)$ \\
\hline \multicolumn{2}{|c|}{ Abortion method on this occasion } \\
\hline Medical & $1449(22.8 \%)$ \\
\hline Surgical & $4892(77.1 \%)$ \\
\hline Both medical and surgical & $7(0.1 \%)$ \\
\hline
\end{tabular}

collection form was completed at the time of the woman's discharge from the service and was anonymous, with no individually identifying information recorded.

Demographic information recorded included age, postcode, relationship status, country of birth, number of children and number of previous terminations. Staff documented the method of abortion used - medical

2 Type of post-abortion contraception chosen*

\begin{tabular}{lcc} 
& No. & $\%(95 \% \mathrm{Cl})$ \\
\hline Male condom & 965 & $15.2 \%(11.1 \%-19.3 \%)$ \\
Female condom & 16 & $0.3 \%(0.1 \%-0.4 \%)$ \\
Combined oral contraceptive pill & 2442 & $38.5 \%(35.4 \%-41.5 \%)$ \\
Vaginal ring & 38 & $0.6 \%(0.3 \%-0.9 \%)$ \\
Progesterone-only pill & 90 & $1.4 \%(0.7 \%-2.1 \%)$ \\
Injection (eg, Depo-Provera) & 238 & $3.7 \%(3.1 \%-4.4 \%)$ \\
Implant (eg, Implanon NXT) & 761 & $12.0 \%(10.6 \%-13.4 \%)$ \\
Intrauterine device (eg, copper) & 173 & $2.7 \%(1.7 \%-3.8 \%)$ \\
Intrauterine system (eg, Mirena) & 810 & $12.8 \%(9.3 \%-16.2 \%)$ \\
Natural methods & 16 & $0.3 \%(0.1 \%-0.4 \%)$ \\
Withdrawal method & 10 & $0.2 \%(0 \%-0.3 \%)$ \\
None & 721 & $11.4 \%(5.9 \%-16.8 \%)$ \\
Vasectomy & 40 & $0.6 \%(0.4 \%-0.9 \%)$ \\
Tubal ligation & 132 & $2.1 \%(1.5 \%-2.7 \%)$ \\
\hline * Some women reported choosing more than one method. If they reported any of Natural, \\
Withdrawal or None, and also reported amedical” method they were classified as only reporting \\
the medical method.
\end{tabular}

abortion, surgical abortion or surgical abortion after a failed medical abortion. The staff recorded the type of post-abortion contraception chosen and whether it was put in place before discharge from the service.

Ethics approval was granted by the University of Sydney Human Research Ethics Committee, and was in keeping with the guidelines set forth by the National Health and Medical Research Council's National Statement on Ethical Conduct in Research Involving Humans.

\section{Statistical analysis}

We calculated the frequency and percentage of women in each subcategory of the demographic characteristics - age, postcode (grouped into Socio-Economic Index for Areas [SEIFA] quintiles using the Australian Bureau of Statistics Index of Relative Socio-Economic Advantage and Disadvantage) ${ }^{20}$ relationship status, country of birth, number of children and number of previous terminations. We examined the association between the choice of a LARC (IUC or implants) after abortion and the demographic characteristics using logistic regression. Also, among women who chose a LARC after abortion, we examined the association between having it inserted at the time of the abortion and the demographic characteristics using logistic regression. In both models, we adjusted for clustering by clinic and stratification by state. Ninety-five per cent confidence intervals for the proportion of women who selected a particular contraceptive method after abortion were also calculated, adjusting for clinic and state. All analyses were carried out with SAS 9.3 (SAS Institute Inc, 2011).

\section{Results}

Between September and December 2012, data were collected from 16 centres across five states and territories. The audit form completion rate in each centre varied from $22.7 \%$ to $95.3 \%$. In total, 7267 of the 9477 women $(76.7 \%)$ who presented during the study period were included in the study. There were 7162 women (98.6\%) who had complete data recorded for all the demographic variables (excluding postcode) and choice of contraception after abortion, and of these women, 6348 (88.6\%) had a postcode that could be matched to a SEIFA quintile.

The demographic characteristics of these women are presented in Box 1. The largest proportion of women was in the 30-39 year age group, followed by those aged $20-24$ years. Two-thirds (4083; 64.3\%) were Australian born, with many other smaller countries of origin represented. Half the sample was nulliparous. Almost two-thirds (3933 of $6348 ; 62.0 \%$ ) had no history of abortion but 725 (11.4\%) had a history 
3 Association of demographic and reproductive factors with choice of long-acting reversible contraception (LARC) after abortion

\begin{tabular}{|c|c|c|c|}
\hline & $\begin{array}{l}\text { Women who chose } \\
\text { LARC (\%)* }\end{array}$ & $\begin{array}{l}\text { Adjusted odds ratio } \\
(95 \% \mathrm{Cl})\end{array}$ & $P$ \\
\hline \multicolumn{3}{|l|}{ Age group (years) } & $<0.001$ \\
\hline Under 16 & $22(48.9 \%)$ & $2.98(1.75-5.10)$ & \\
\hline $16-19$ & $202(28.9 \%)$ & $1.31(1.12-1.52)$ & \\
\hline $20-24$ & $456(26.4 \%)$ & 1.00 (reference) & \\
\hline $25-29$ & $383(25.4 \%)$ & $0.75(0.63-0.90)$ & \\
\hline 30-39 & $523(27.2 \%)$ & $0.61(0.48-0.77)$ & \\
\hline 40 and over & $156(35.1 \%)$ & $0.78(0.56-1.07)$ & \\
\hline \multicolumn{3}{|l|}{ Relationship status } & 0.42 \\
\hline Single & $723(26.4 \%)$ & $0.96(0.85-1.07)$ & \\
\hline Partnered & $1019(28.3 \%)$ & 1.00 (reference) & \\
\hline \multicolumn{3}{|l|}{ Birthplace } & $<0.001$ \\
\hline Australia & $1184(29.0 \%)$ & 1.00 (reference) & \\
\hline Other Pacific nation & $123(29.1 \%)$ & $0.95(0.7-1.28)$ & \\
\hline Asia & $244(21.8 \%)$ & $0.72(0.65-0.81)$ & \\
\hline Africa & $50(31.1 \%)$ & 1.09 (0.87-1.37) & \\
\hline America (North and South) & $19(24.7 \%)$ & $0.93(0.63-1.35)$ & \\
\hline Europe & $93(28.4 \%)$ & $1.11(0.83-1.47)$ & \\
\hline Middle East & $29(18.1 \%)$ & $0.57(0.37-0.90)$ & \\
\hline \multicolumn{3}{|c|}{ Socio-Economic Index for Areas quintile } & 0.12 \\
\hline 1 (most disadvantaged) & $255(25.7 \%)$ & 1.00 (reference) & \\
\hline 2 & $271(30.7 \%)$ & $1.24(1.02-1.52)$ & \\
\hline 3 & $436(29.3 \%)$ & $1.23(1.00-1.52)$ & \\
\hline 4 & $347(28.1 \%)$ & $1.24(0.94-1.65)$ & \\
\hline 5 (least disadvantaged) & $433(24.7 \%)$ & $1.13(0.88-1.45)$ & \\
\hline \multicolumn{3}{|l|}{ Number of children } & $<0.001$ \\
\hline 0 & $665(20.9 \%)$ & 1.00 (reference) & \\
\hline 1 & $336(29.6 \%)$ & $1.92(1.71-2.15)$ & \\
\hline 2 & $461(36.0 \%)$ & $2.88(2.24-3.69)$ & \\
\hline 3 & $181(36.9 \%)$ & $2.93(2.24-3.83)$ & \\
\hline 4 or more & $99(38.8 \%)$ & $3.3(2.19-4.95)$ & \\
\hline \multicolumn{3}{|c|}{ Number of previous terminations } & 0.095 \\
\hline 0 & $995(25.3 \%)$ & 1.00 (reference) & \\
\hline 1 & $521(30.8 \%)$ & $1.23(1.05-1.44)$ & \\
\hline 2 & $150(29.6 \%)$ & $1.07(0.85-1.35)$ & \\
\hline 3 & $52(35.1 \%)$ & $1.34(0.79-2.27)$ & \\
\hline 4 or more & $24(33.8 \%)$ & $1.23(0.62-2.42)$ & \\
\hline \multicolumn{3}{|c|}{ Abortion method on this occasion } & 0.089 \\
\hline Medical & $342(23.6 \%)$ & 1.00 (reference) & \\
\hline Surgical & $1398(28.6 \%)$ & $1.16(0.98-1.38)$ & \\
\hline Both medical and surgical ${ }^{\dagger}$ & $2(28.6 \%)$ & & \\
\hline
\end{tabular}

of two or more previous terminations of pregnancy.

\section{Contraceptive choices after abortion}

The most popular contraceptive method adopted after abortion was the oral contraceptive pill, chosen by more than a third of women $(2442$ of $6348 ; 38.5 \%$ ) (Box 2). Few women elected to take the progesterone-only

by $981(15.5 \%)$. Half the women chose one of the user-dependent modern methods (3551; 56.0\%). Around one woman in 10 elected not to use any contraception (721; 11.4\%). Of the 2532 women who chose either of the pills, $2296(90.7 \%)$ had a prescription provided after abortion (95\% CI, 87.5\%93.9\%). Injectable progestogen was taken up by 238 women (3.7\%) and just over a quarter of women chose either an implant or IUC LARC method (1742; 27.4\%).

\section{LARC choice after abortion}

Choice of LARC was influenced by age, with younger women $(<20$ years) more likely to choose a LARC method compared with older women (Box 3). Women born in Australia were more likely than Asian- and Middle Eastern-born women to opt for a LARC method (adjusted odds ratio [AOR], 0.72; 95\% CI, 0.65-0.81, and 0.57; 95\% CI, 0.37-0.90, respectively).

LARC method choice was associated with number of children, with the likelihood of LARC choice increasing with number of children. There was no association between type of abortion and choosing a LARC method.

\section{Insertion of LARC after abortion}

Of the 1742 women who chose a LARC method, 1238 (71.1\%) had immediate provision of their implant or IUC at the time of surgical abortion or, in the case of medical abortion, at the 2-week follow-up visit. Thus, overall, $19.5 \%$ left the service with their LARC method in place. Among women who opted for a LARC, the actual provision of LARC immediately after abortion was significantly more common after surgical abortion compared with medical abortion (AOR, 2.26; 95\% CI, 1.58-3.24) (Box 4). Immediate insertion occurred more frequently among women aged over 30 compared with younger women, and among women who were Australian or African born. In a test for trend, women were more likely to receive a LARC method as their level of disadvantage lessened $(P<0.026)$. Women who had had one previous termination were significantly more likely to leave the service with the LARC method in place, compared with women who had presented for their first abortion (AOR, 1.37; 95\% CI, 1.11-1.70).

\section{Discussion}

This study documented Australian women's choice of post-abortion contraception, with a particular focus on the uptake of the most reliable methods of contraception, implants and IUC. We found that, despite a 
4 Association of demographic and reproductive factors with long-acting reversible contraception (LARC) insertion immediately after abortion

\begin{tabular}{|c|c|c|c|}
\hline & $\begin{array}{l}\text { Women who had LARC } \\
\text { inserted immediately, no. } \\
(\%)^{*}\end{array}$ & $\begin{array}{l}\text { Adjusted odds } \\
\text { ratio }(95 \% \mathrm{Cl})\end{array}$ & $P$ \\
\hline Age group (years) & & & 0.007 \\
\hline Under 16 & $16(72.7 \%)$ & $1.35(0.46-3.96)$ & \\
\hline $16-19$ & $129(64.5 \%)$ & $0.81(0.51-1.27)$ & \\
\hline $20-24$ & $309(68.2 \%)$ & 1.00 (reference) & \\
\hline $25-29$ & $262(68.8 \%)$ & $1.06(0.74-1.51)$ & \\
\hline $30-39$ & $394(75.6 \%)$ & $1.47(1.03-2.09)$ & \\
\hline 40 and over & $128(82.6 \%)$ & $2.24(1.22-4.11)$ & \\
\hline Relationship status & & & 0.98 \\
\hline Single & $508(70.7 \%)$ & $1.00(0.80-1.24)$ & \\
\hline Partnered & $730(72.1 \%)$ & 1.00 (reference) & \\
\hline Birthplace & & & $<0.001$ \\
\hline Australia & $830(70.6 \%)$ & 1.00 (reference) & \\
\hline Other Pacific nation & $73(59.4 \%)$ & $0.6(0.44-0.81)$ & \\
\hline Asia & $183(75.3 \%)$ & $1.13(0.83-1.54)$ & \\
\hline Africa & $42(84.0 \%)$ & $2.34(1.22-4.49)$ & \\
\hline America (North and South) ${ }^{\dagger}$ & $19(100.0 \%)$ & & \\
\hline Europe $^{\dagger}$ & $72(77.4 \%)$ & $1.7(0.99-2.93)$ & \\
\hline Middle East & $19(67.9 \%)$ & $0.97(0.33-2.87)$ & \\
\hline Socio-Economic Index for Areas qu & uintile & & $0.026 \ddagger$ \\
\hline 1 (most disadvantaged) & $168(66.7 \%)$ & 1.00 (reference) & \\
\hline 2 & $185(68.8 \%)$ & $1.17(0.87-1.57)$ & \\
\hline 3 & $311(71.5 \%)$ & $1.26(0.90-1.77)$ & \\
\hline 4 & $252(72.8 \%)$ & $1.50(0.97-2.32)$ & \\
\hline 5 (least disadvantaged) & $322(74.9 \%)$ & $1.54(1.04-2.26)$ & \\
\hline Number of children & & & 0.19 \\
\hline 0 & $454(68.9 \%)$ & 1.00 (reference) & \\
\hline 1 & $234(79.9 \%)$ & $0.89(0.68-1.16)$ & \\
\hline 2 & $346(75.2 \%)$ & $1.01(0.73-1.39)$ & \\
\hline 3 & $135(75.4 \%)$ & $1.04(0.74-1.46)$ & \\
\hline 4 or more & $69(69.7 \%)$ & $0.65(0.34-1.27)$ & \\
\hline Number of previous terminations & & & $<0.001$ \\
\hline 0 & $686(69.3 \%)$ & 1.00 (reference) & \\
\hline 1 & $395(76.1 \%)$ & $1.37(1.11-1.70)$ & \\
\hline 2 & $105(71.0 \%)$ & $0.95(0.72-1.27)$ & \\
\hline 3 & $33(64.7 \%)$ & $0.72(0.40-1.28)$ & \\
\hline 4 or more & $19(79.2 \%)$ & $1.65(0.59-4.58)$ & \\
\hline Abortion method on this occasion & & & $<0.001$ \\
\hline Medical & $204(60.0 \%)$ & 1.00 (reference) & \\
\hline Surgical & $1034(74.4 \%)$ & $2.26(1.58-3.24)$ & \\
\hline Both medical and surgical & 0 & & \\
\hline
\end{tabular}

*Row percentages. Data on timing of LARC insertion were missing for 10 women, who were therefore excluded from the analysis. $\dagger$ America (North and South) was grouped with Europe for analysis, as all women within the America (North and South) category had a LARC inserted. $¥ P$ for quintiles is test for trend.

history of previous abortion in $38.0 \%$ of the sample, under a third of women attending one of the MSIA clinics indicated their intention to use one of these LARC methods after abortion, and in only one in five was the method inserted immediately. Based on population data, this rate of LARC
3.30; 95\% CI, 2.67-4.85) and 50\% more likely to choose the implant (adjusted RR, 1.51; 95\% CI, 1.12-2.03) compared with women without a recent abortion. ${ }^{22}$ Importantly, these methods are potentially of greatest benefit to a population at risk of repeated unintended pregnancy. In a Scottish study of 986 women who sought abortion, $121(12.3 \%)$ returned requesting another abortion within the subsequent 2 years. The chance of repeat abortion was reduced by $95 \%$ in women previously provided either IUC or the implant compared with women prescribed the oral contraceptive pill. ${ }^{14}$

In our study, several demographic factors were associated with greater likelihood of LARC choice, including young age and having one or more child. Proportionally fewer women who were Asian- and Middle Eastern-born chose a LARC method compared with Australian-born women. In contrast, immediate LARC provision was associated with women aged 30 years and older, and least likely to occur in younger age groups. Socioeconomic status, as measured by SEIFA quintiles, was linearly associated with LARC provision before discharge, with those women living in areas of highest disadvantage least likely to have a LARC method inserted.

The difference between stated intention to use a LARC and actual immediate provision may reflect the fact that women are required to pay an additional fee to have a LARC method initiated at the time of surgical and medical abortion. While the cost of the implant and the hormonal IUC are both subsidised by the Pharmaceutical Benefits Scheme, the copper IUC is not. Some women, particularly those who are younger or from areas of high socioeconomic disadvantage, may have faced difficulty finding the extra money required for upfront payment of their chosen LARC method. Although MSI use telephone health interpreter services for women of non-English speaking background, language may have been a barrier to contraceptive information provision for some women.

The type of abortion chosen, while not associated with LARC choice, was associated with LARC provision with greater likelihood of initiation after 
surgical abortion compared with medical abortion. Women may have seen a benefit in having the LARC method (especially an IUC) inserted while under sedation, as is commonly practised with surgical abortion.

There are, however, additional challenges to ensuring this transpires after medical abortion, as it requires clinicians to be certain the abortion is complete and to be available soon after expulsion of the pregnancy has occurred. The evidence supports insertion of IUC within 5 to 9 days of medical abortion, and it has been found to be as safe as delayed insertion at 3-4 weeks with no increased rate of expulsions or complications. ${ }^{19}$ Delayed insertion of the LARC, although sometimes unavoidable, may mean that the method is never initiated, with one study showing that $40 \%$ of clients did not return for the insertion of their IUC. ${ }^{18}$

This study represents a large number of women. Nevertheless, we sampled only $76.7 \%$ of those presenting. The audit forms were completed by nursing and medical staff. Noncompleted forms are more likely to represent systemic flaws at the clinic level rather than the individual level, as some centres only completed forms for a minority of clients. Although we were able to document the factors associated with LARC uptake and non-uptake, we were not able to explore in depth what the key barriers perceived by women to be to adopting these methods were.

While abortion services recognise the need to ensure women leave their services with reliable contraception, there needs to be greater emphasis on ensuring that LARC methods are made more accessible and more affordable. This will enable more women to avoid a further unintended pregnancy.

Competing interests: Kirsten Black is a consultant on an international advisory board for Bayer HealthCare, and has been supported in this capacity to attend conferences as a presenting author; she has also received payment as a speaker. She is a registered trainer in implant insertion and removal for MSD. Philip Goldstone is a registered trainer in implant insertion and removal for MSD.

Received 3 Jan 2014, accepted 21 May 2014

1 Sexual and Reproductive Health Strategy Reference Group. Time for a national sexual and reproductive health strategy for Australia. Public Health Association of Australia, Sexual Health \& Family Planning Association of Australia, Australian Reproductive Health Alliance, 2008. http://www.fpwa.org.au/ resources/srhbackground.pdf (accessed Jan 2014)

2 Marie Stopes International Australia. Real choices: women, contraception and unplanned pregnancy. Sydney: MSIA, 2008.

3 Chan A, Sage LC. Estimating Australia's abortion rates 1985-2003. Med J Aust 2005 182: 447-452.

4 Lucke J, Herbert D, Loxton D, Weisberg E. Unintended pregnancies: reducing rates by improving access to contraception. Aust Fam Physician 2011; 40: 849

5 Sexual Health and Family Planning Australia. Time for a change: increasing the use of long acting reversible contraception methods in Australia. SH\&FPA, 2013. http://www.fpnsw. org.au/larc.pdf (accessed Jan 2014)
6 National Collaborating Centre for Women's and Children's Health. Longacting reversible contraception: the effective and appropriate use of longacting reversible contraception. London: RCOG Press, 2005. http://www.nice. org.uk/nicemedia/live/10974/29912/29912.pdf (accessed Jan 2014).

7 American College of Obstetricians and Gynecologists. ACOG Practice Bulletin No. 121: long-acting reversible contraception: implants and intrauterine devices. Obstet Gynecol 2011; 118: 184-196.

8 Winner B, Peipert JF, Zhao Q, et al. Effectiveness of long-acting reversible contraception. NEngl J Med 2012; 366: 1998-2007.

9 Gray E, McDonald P. Using a reproductive life course approach to understand contraceptive method use in Australia. J Biosoc Sci 2010; 42: 43-57.

10 Mazza D, Harrison C, Taft A, et al. Current contraceptive management in Australian general practice: an analysis of BEACH data. Med J Aust 2012; 197: $110-114$.

11 Rose SB, Lawton BA. Impact of long-acting contraception on return for repeat abortion. Am J Obstet Gynecol 2012; 206: el-e6.

12 Roberts H, Silva M, Xu S. Post abortion contraception and its effect on repeat abortions in Auckland, New Zealand. Contraception 2010; 82: 260-265.

13 Goodman S, Hendlish SK, Reeves MF, Foster-Rosales A. Impact of immediate postabortal insertion of intrauterine contraception on repeat abortion. Contraception 2008; 78: 143-148.

14 Cameron ST, Glasier A, Chen ZE, et al. Effect of contraception provided at termination of pregnancy and incidence of subsequent termination of pregnancy. BJOG 2012; 119: 1074-1080.

15 Royal College of Obstetricians and Gynaecologists. The care of women requesting induced abortion. London: RCOG, 2011.

16 World Health Organization. Safe abortion: technical and policy guidance for health systems. Geneva: WHO, 2012

17 Lahteenmäki P, Luukkainen T. Return of ovarian function after abortion. Clin Endocrinol (Oxf) 1978; 8: 123-132.

18 Gillett PG, Lee NH, Yuzpe AA, Cerskus I. A comparison of the efficacy and acceptability of the Copper-7 intrauterine device following immediate or delayed insertion after first-trimester therapeutic abortion. Fertil Steril 1980; 34: 121-124.

19 Sääv I, Stephansson 0, Gemzell-Danielsson K. Early versus delayed insertion of intrauterine contraception after medical abortion - a randomized controlled trial. PLOS One 2012; 7: e48948.

20 Australian Bureau of Statistics. Socio-economic Indexes for Areas - Technica Paper, 2011. Canberra: ABS, 2011. (ABS Cat. No. 2033.0.55.001.) http://www. abs.gov.au/ausstats/abs@.nsf/mf/2033.0.55.001 (accessed Jan 2014).

21 Richters J, Grulich A, de Visser Ro, et al. Sex in Australia: contraceptive practices among a representative sample of women. Aust N Z J Public Health 2003; 27: 210-216.

22 Madden T, Secura GM, Allsworth JE, Peipert JF. Comparison of contraceptive method chosen by women with and without a recent history of induced abortion. Contraception 2011; 84: 571-577. 